\title{
The Structure of the Quasi-invariant Set of a Linear Measure
}

\author{
By \\ Yoshiaki OKAZAKI* and Yasuji TAKAHASHI**
}

\begin{abstract}
Let $\mu$ be a probability measure on a locally convex Hausdorff space $E$ and $A(\mu)$ be the quasi-invariant set of $\mu$. If $\mu^{*}(A(\mu))>0$, then there exist a finite-dimensional subspace $L$, a thick subgroup $G$ of $L$ and a countable subgroup $\left\{x_{i}\right\}$ such that $A(\mu)=$ $\bigcup_{i=1}^{\infty}\left(G+x_{i}\right)$. If $E$ is Souslin, then $A(\mu)$ is a Borel subset. If $E$ is Souslin and if $\mu(A(\mu))$ $>0$, then $A(\mu)=\bigcup_{i=1}^{\infty}\left(L+x_{i}\right)$.
\end{abstract}

\section{$\S 1$. Introduction}

Let $E$ be a locally convex Hausdorff space and $E^{\prime}$ be the topological dual of $E$. Denote by $C\left(E, E^{\prime}\right)$ the cylindrical $\sigma$-algebra on $E$, the minimal $\sigma$-algebra which makes each $\left\langle, x^{\prime}\right\rangle, x^{\prime} \in E^{\prime}$, measurable. Denote by $B(E)$ the Borel $\sigma$-algebra on $E$ generated by all open subsets. Then it holds that $C\left(E, E^{\prime}\right) \subset B(E)$ and these $\sigma$-algebras are translation invariant, that is, for every $x \in E$ and $A \in$ $C\left(E, E^{\prime}\right)$ (resp. $B(E)$ ) it follows that $A-x \in C\left(E, E^{\prime}\right)$ (resp. $B(E)$ ). Let $\mu$ be a probability measure on $C\left(E, E^{\prime}\right)$ or on $B(E)$. For $x \in E$ we set $\mu_{x}(A)=\mu(A-x)$ and $A(\mu)=\left\{x \in E: \mu_{x} \sim \mu\right.$ (equivalent) $\}$. The set $A(\mu)$ is called the quasi-invariant set of $\mu$. It is well-known that $A(\mu)$ is an additive subgroup of $E$.

Skorohod [9] stated the following assertions concerning the structure of the quasi-invariant set $A(\mu)$ (however there are some gaps in the proof).

Let $E$ be a separable Hilbert space. Then

(1) $A(\mu)$ is a Borel subset of $E$,

(2) if $\mu(F)=0$ for every finite-dimensional subspace $F \subset E$, then $\mu(A(\mu))=0$, and

(3) if $\mu(A(\mu))=1$, then there exists a sequence $L_{i}$ of finitedimensional subspaces such that $\mu\left(\bigcup_{i=1}^{\infty} L_{i}\right)=1$,

see [9], § 19. On the other hand, Okazaki [7] proved the following results.

Communicated by S. Matsuura, April 27, 1990.

* Department of Artificial Intelligence, Kyushu Inst. of Technology, Iizuka 820, Japan.

** School of Health Sciences, Okayama University, Okayama 700, Japan. 
Let $G$ be a complete separable metrizable abelian topological group and $\mu$ be a probability measure on $G$. Then

(1) $A(\mu)$ is a Borel subset of $G$, and

(2) if $\mu(A(\mu))>0$, then $A(\mu)$ is a locally compact $\sigma$-compact topological group with respect to the induced topology from $G$.

There are similarities between these two results. In fact, each locally compact locally convex Hausdorff space is finite-dimensional and each locally compact $\sigma$ compact subgroup of a locally convex Hausdorff space is of the form $R^{n}+$ (countable subgroup) (the structure theorem of the locally compact $\sigma$-compact abelian topological group).

In this paper, we generalize the above results of Skorohod as follows. Firstly, suppose that $E$ is a general locally convex Hausdorff space and $\mu^{*}(A(\mu))$ $>0$, then there exist a finite-dimensional subspace $L$, a thick subgroup $G$ of $L$ and a countable subgroup $\left\{x_{i}\right\}$ of $E$ such that $A(\mu)=\bigcup_{i=1}^{\infty}\left(G+x_{i}\right)$ (Theorem 1), where $\mu^{*}$ denotes the outer measure. Secondly, suppose that $E$ is a Souslin locally convex Hausdorff space, then $A(\mu)$ is a Borel subset of $E$ and if $\mu(A(\mu))$ $>0, A(\mu)$ can be written as $A(\mu)=\bigcup_{i=1}^{\infty}\left(L+x_{\imath}\right)$, where $L$ is a finite-dimensional subspace and $\left\{x_{i}\right\}$ is a countable subgroup of $E$ (Theorem 2).

\section{§2. Preliminaries}

Let $(G, B)$ be a measurable group, that is, $G$ is a group with a $\sigma$-algebra $B$ satisfying

(1) $x \longrightarrow x^{-1}$ is $B$-measurable, and

(2) $(x, y) \longrightarrow x y$ is $B \otimes B-B$-measurable, where $B \otimes B$ is the product $\sigma$-algebra on $G \times G$, see Halmos [4], § 59 and Yamasaki [12], Part B, Chapter $1, \S 1$.

Let $(G, B)$ be a measurable group and $\mu$ be a measure on $(G, B)$. Then $(G, B, \mu)$ is called separated if for every $g \neq e$ ( $e$ is the unit of $G$ ), there exists $A \in B$ such that $\mu(A)>0$ and $\mu(A \cap A g)=0$, see Halmos [4], $\S 62$ and Yamasaki [12], Part B, Chapter 1, §4.

A subset $A$ of a topological group $G$ is called precompact (or bounded) if for every neighborhood $U$ of $e$, there exists a finite sequence $g_{1}, g_{2}, \cdots, g_{n}$ in $G$ such that $A \subset \bigcup_{\imath=1}^{n}\left(U g_{\imath}\right)$. If a topological group $G$ has a precompact neighborhood of $e$, then $G$ is called locally precompact. If a topological group $G$ is written as $G=\bigcup_{n=1}^{\infty} A_{n}$, where each $A_{n}$ is precompact, then $G$ is called $\sigma$-precompact, see Halmos [4], §0 and Yamasaki [12], Part B, Chapter 1, §3.

We shall use the following facts in later. 
Fact 1. A topological group $G$ is locally precompact and $\sigma$-precompact if and only if $G$ can be imbedded densely and isomorphically (algebraically and topologically isomorphic) into a locally compact $\sigma$-compact topological group $\bar{G}$.

The group $\bar{G}$ is uniquely determined within an isomorphism and $\bar{G}$ is called the completion of $G$, see Bourbaki [1], Chapter 2, §3, Halmos [4], §0 and Yamasaki [12], Part B, Chapter 1, §3, Theorem 3.1. As for the Borel structure of $G$ and $\bar{G}$, the following result is known, see Yamasaki [12], Part B, Chapter $1, \S 3$, Theorem 3.2.

Fact 2. Let $G$ be a locally precompact $\sigma$-precompact topological group and $B u$ be the $\sigma$-algebra on $G$ generated by all uniformly continuous functions on $G$. Let $\overline{B a}$ be the Baire $\sigma$-algebra on the completion $\bar{G}$, the $\sigma$-algebra generated by all continuous functions on $\bar{G}$. Then it holds that $B u=\overline{B a} \cap G$. Moreover, $(G, B u)$ is a measurable group.

A topological group $G$ is called a thick group if

(1) $G$ is locally precompact and $\sigma$-precompact, and

(2) for the right Haar measure $\bar{\lambda}$ on $(\bar{G}, \overline{B a}), G$ is thick with respect to $\bar{\lambda}$, that is, for every $B \in \overline{B a}$ with $B \cap G=\phi$ it follows that $\bar{\lambda}(B)=0$,

see Halmos [4], §62 and Yamasaki [12], Part B, Chapter 1, §3.

Let $G$ be a thick group. Then there is a right invariant measure $\lambda$ on $(G, B u)$, that is, $\lambda(A g)=\lambda(A)$ for every $A \in B u$ and every $g \in G$. In fact, $\lambda$ is the restriction of $\bar{\lambda}$ to $G$, see Yamasaki [12], Part B, Chapter $1, \S 3$, Theorem 3.3. This right invariant measure $\lambda$ on $(G, B u)$ is called the Haar measure of $G$. It is known that, in general, a right invariant measure on $(G, B u)$ is unique up to a constant factor.

In the sequel, every group which we consider is abelian. Hence we derote by $x+y$ the group operation (instead of $x y$ ).

\section{§3. Quasi-invariant Set for Cylindrical Measure}

Let $E$ be a locally convex Hausdorff space and $\mu$ be a probability measure on $C\left(E, E^{\prime}\right)$. Then $\left(E, C\left(E, E^{\prime}\right)\right)$ is a measurable group and the quasi-invariant set $A(\mu)$ is an additive subgroup of $E$, see Yamasaki [12], Part B, Chapter $1, \S 5$, Theorem 5.1. Remark also that $C\left(E, E^{\prime}\right) B C\left(E, E^{\prime}\right)=C\left(E \times E,(E \times E)^{\prime}\right)$. The next lemma is an easy consequence of this fact.

Lemma 1. $\left(A(\mu), C\left(E, E^{\prime}\right) \cap A(\mu)\right)$ is a measurable group.

Proof. We show that $\Psi:(x, y) \longrightarrow x-y$ is $\beta \otimes \beta-\beta$-measurable, where $\beta=$ $C\left(E, E^{\prime}\right) \cap A(\mu)$. For every $B \in \beta$, we can find $C$ so that $B=C \cap A(\mu)$ and $C \in$ $C\left(E, E^{\prime}\right)$. Then we have $\Psi^{-1}(B)=\Psi^{-1}(C) \cap A(\mu) \times A(\mu) \in C\left(E, E^{\prime}\right) \otimes C\left(E, E^{\prime}\right) \cap$ 
$A(\mu) \times A(\mu)=\beta \otimes \beta$.

Suppose that $\mu^{*}(A(\mu))>0$ where $\mu^{*}$ is the outer measure. Let $\nu$ be the restriction of $\mu^{*}$ to $\left(A(\mu), C\left(E, E^{\prime}\right) \cap A(\mu)\right)$. $\nu$ is defined as follows. Take $C \in$ $C\left(E, E^{\prime}\right)$ such that $A(\mu) \subset C$ and $\mu(C)=\mu^{*}(A(\mu))$. Then for every $A \in C\left(E, E^{\prime}\right)$ $\cap A(\mu)$ with $A=D \cap A(\mu), D \in C\left(E, E^{\prime}\right), \nu(A)$ is given by $\nu(A)=\mu(C \cap D)$.

Lemma 2. Suppose that $\mu^{*}(A(\mu))>0$ and $\nu$ be the restriction of $\mu^{*}$ to $(A(\mu)$, $\left.C\left(E, E^{\prime}\right) \cap A(\mu)\right)$. Then $\nu$ is a quasi-invariant measure on $A(\mu)$, that is, $\nu$ and $\nu_{x}$ are equivalent for every $x \in A(\mu)$.

Proof. Let $C \in C\left(E, E^{\prime}\right)$ be $A(\mu) \subset C$ and $\mu^{*}(A(\mu))=\mu(C)$. For every $A \in$ $C\left(E, E^{\prime}\right) \cap A(\mu)$, we write $A=D \cap A(\mu)$, where $D \in C\left(E, E^{\prime}\right)$. Then we have for each $x \in A(\mu), A-x=(D-x) \cap A(\mu)$ and $A(\mu) \subset(C-x) \cap C \subset C$. Hence it follows that $\mu(C-x)=\mu(C)=\mu^{*}(A(\mu))$ and $\nu_{x}(A)=\nu(A-x)=\nu((D-x) \cap A(\mu))=\mu(C \cap(D-x))$ $=\mu((C-x) \cap(D-x))=\mu(C \cap D-x)=\mu_{x}(C \cap D)$ for every $x \in A(\mu)$. Since $\nu(A)=$ $\mu(C \cap D)$, for every $x \in A(\mu), \nu_{x}(A)=0$ if and only if $\nu(A)=0$.

Lemma 3. Suppose that $\mu^{*}(A(\mu))>0$ and $\nu$ be the restriction of $\mu^{*}$ to $(A(\mu)$, $\left.C\left(E, E^{\prime}\right) \cap A(\mu)\right)$. Then $\left(A(\mu), C\left(E, E^{\prime}\right) \cap A(\mu), \nu\right)$ is separated.

Proof. For every $x \neq 0$ in $A(\mu)$, we shall show the existence of $A$ in $C\left(E, E^{\prime}\right) \cap A(\mu)$ such that $\nu(A)>0$ and that $A \cap(A+x)=\phi$. Let $x^{\prime} \in E^{\prime}$ be $\left\langle x, x^{\prime}\right\rangle$ $=1$ (Hahn-Banach theorem) and set $A=\left\{y \in A(\mu): 0 \leqq\left\langle y, x^{\prime}\right\rangle<1\right\}$. Then it holds that $A \cap(A+x)=\phi$ and $A(\mu)=\bigcup_{n=1}^{\infty}(A+n x)$. By Lemma 2 , it must be $\nu(A)>0$.

Lemma 4. Suppose that $\mu^{*}(A(\mu))>0$ and $\nu$ be the restriction of $\mu^{*}$ to $(A(\mu)$, $\left.C\left(E, E^{\prime}\right) \cap A(\mu)\right)$. Then there exists a ( $\sigma$-finite by (1) below) measure $\lambda$ on $(A(\mu)$, $\left.C\left(E, E^{\prime}\right) \cap A(\mu)\right)$ such that

(1) $\lambda \sim \nu$ (equivalent), and

(2) $\lambda_{x}=\lambda$ for each $x$ in $A(\mu)(A(\mu)$-invariant).

Proof. The assertions follow by Mackey [6], Lemma 7, Umemura [10], Proposition 6.2 and Yamasaki [12], Part B, Chapter 1, § 1, Theorems 1.4 and 1.1.

Suppose that $\mu^{*}(A(\mu))>0$ and $\nu$ be the restriction of $\mu^{*}$ to $\left(A(\mu), C\left(E, E^{\prime}\right)\right.$ $\cap A(\mu)$ ). Let $\lambda$ be a $\sigma$-finite invariant measure on $A(\mu)$ equivalent to $\nu$ (Lemma 4). We remark that $\left(A(\mu), C\left(E, E^{\prime}\right) \cap A(\mu), \lambda\right)$ is also separated since so is $\left(A(\mu), C\left(E, E^{\prime}\right) \cap A(\mu), \nu\right)$ and $\lambda$ is equivalent to $\nu$.

Now let $\tau$ be the Weil topology of $A(\mu)$ derived by the invariant measure $\left(A(\mu), C\left(E, E^{\prime}\right) \cap A(\mu), \lambda\right)$, that is, the basis of neighborhoods of 0 in $\tau$ is given by the family

$$
U_{A, \varepsilon}=\{x \in A(\mu): \lambda(A \ominus(A+x))<\varepsilon\},
$$

where $A \in C\left(E, E^{\prime}\right) \cap A(\mu)$ be $0<\lambda(A)<\infty, \varepsilon>0$ and $\ominus$ is the symmetric dif- 
ference, see Halmos [4], § 62, Yamasaki [12], Part B, Chapter 1, §4 and Weil [11], Appendice 1. Then the following result is known. For the proof, we refer to Halmos [4], §62 and Yamasaki [12], Part B, Chapter 1, §4, Theorem 4.1.

Lemma 5. $(A(\mu), \tau)$ is a Hausdorff topological group and thick. Moreover, $B u \subset C\left(E, E^{\prime}\right) \cap A(\mu)$ and the restriction $\lambda \mid B u$ is the Haar measure on $(A(\mu), B u)$.

Lemma 6. The Weil topology $\tau$ is finer than the weak topology $\sigma\left(E, E^{\prime}\right)$ on $A(\mu)$. In particular it holds that $B u=C\left(E, E^{\prime}\right) \cap A(\mu)$ in Lemma 5.

Proof. We show that each $x^{\prime}$ is $\tau$-continuous. Let $N$ be $\lambda(\{x \in A(\mu)$ : $\left.\left.\left|\left\langle x, x^{\prime}\right\rangle\right| \leqq N\right\}\right)>0$ and let $A \subset\left\{x \in A(\mu):\left|\left\langle x, x^{\prime}\right\rangle\right| \leqq N\right\}$ be $0<\lambda(A)<\infty$. Then for every $x \in U_{A, \lambda(A)}=\{x \in A(\mu): \lambda(A \Theta(A+x))<\lambda(A)\}$, it follows that $\left|\left\langle x, x^{\prime}\right\rangle\right|$ $\leqq 2 N$ since $U_{A, \lambda(A)} \subset A-A \subset\left\{x \in A(\mu):\left|\left\langle x, x^{\prime}\right\rangle\right| \leqq 2 N\right\}$. Remark that if $\lambda(A \Theta(A+x))<\lambda(A)$ then $A \cap(A+x) \neq \phi$. Thus the additive functional $x^{\prime}$ is $\tau$ continuous.

Theorem 1. Let $E$ be a locally convex Hausdorff space and $\mu$ be a probability measure on $C\left(E, E^{\prime}\right)$. Suppose that $\mu^{*}(A(\mu))>0$. Then

(1) there exists a topology $\tau$ on $A(\mu)$ such that $(A(\mu), \tau)$ is a Hausdorff topological group and a thick group,

(2) the restriction $\nu$ of $\mu^{*}$ to $A(\mu)$ is equivalent to the Haar measure on the thick group $(A(\mu), \tau)$, and

(3) there exist a finite-dimensional subspace $L \subset E$, a thick subgroup $G$ of $L$ (with respect to the Euclidean topology) and a countable subgroup $\left\{x_{i}\right\} \subset$ E such that $A(\mu)=\bigcup_{\imath=1}^{\infty}\left(G+x_{\imath}\right) \subset \bigcup_{\imath=1}^{\infty}\left(L+x_{\imath}\right)$.

Proof. (1) and (2) follow from Lemmas 5 and 6 . We shall prove (3). Consider the natural injection $\iota:(A(\mu), \tau) \rightarrow\left(E, \sigma\left(E, E^{\prime}\right)\right)$. By Lemma 6, $\iota$ is continuous. $c$ can be extended to the completion $(A(\mu), \tau)^{-}$into $\left(E^{\prime}\right)^{a}$ (the algebraic dual of $E^{\prime}$ which is the completion of $\left(E, \sigma\left(E, E^{\prime}\right)\right)$, see Bourbaki [1], Chapter 2 , §3, Theorem 3.1. Let $\bar{c}$ be the extension. Then $\bar{c}$ is a continuous homomorphism on the locally compact $\sigma$-compact topological group $(A(\mu), \tau)^{-}$into $\left(\left(E^{\prime}\right)^{a}, \sigma\left(\left(E^{\prime}\right)^{a}, E^{\prime}\right)\right)$. The image $i\left((A(\mu), \tau)^{-}\right)$is algebraically isomorphic with $(A(\mu), \tau)^{-} / \operatorname{ker} \bar{c}$. We put the topology $T$ on $i\left((A(\mu), \tau)^{-}\right)$induced by the quotient topology of $(A(\mu), \tau)^{-} / \operatorname{ker} \bar{\imath}$. Then $\left(\bar{\imath}\left((A(\mu), \tau)^{-}\right), T\right)$ is again a locally compact $\sigma$-compact abelian topological group and $T$ is finer than the weak topology $\sigma\left(\left(E^{\prime}\right)^{a}, E^{\prime}\right)$. By the structure theorem of locally compact $\sigma$-compact abelian topological group, there exist natural numbers $n, d$ (possibly 0 ) and a compact abelian group $K$ such that $\left(\bar{\imath}\left((A(\mu), \tau)^{-}\right), T\right)$ is isomorphic (algebraically and topologically) with the direct sum $R^{n} \oplus Z^{d} \oplus K$, where $R$ (resp. $Z$ ) denotes the real numbers (resp. integers), see Hewitt and Ross [5], Theorem (9.8) and Weil [11], §29. Since $K$ is isomorphic to a compact subgroup of the vector space $\left(\left(E^{\prime}\right)^{a}, \sigma\left(\left(E^{\prime}\right)^{a}, E^{\prime}\right)\right)$, 
it must be $K=\{0\}$. Let $\phi: R^{n} \oplus Z^{d} \rightarrow\left(\bar{\imath}\left((A(\mu), \tau)^{-}\right), T\right) \subset\left(E^{\prime}\right)^{a}$ be an isomorphism. Then $\psi$ is in fact linear on $R^{n}$, hence $L=\psi\left(R^{n}\right)$ is a finite-dimensional subspace of $\left(E^{\prime}\right)^{a}$ contained in $\bar{\imath}\left((A(\mu), \tau)^{-}\right) . \quad D=\psi\left(Z^{n}\right)$ is a discrete countable subgroup of $\left(\bar{l}\left((A(\mu), \tau)^{-}\right), T\right)$. We have proved that $\left(\bar{l}\left((A(\mu), \tau)^{-}\right), T\right)=L \oplus D$. Remark that $L$ is open and closed subgroup of $\left(\bar{l}\left((A(\mu), \tau)^{-}\right), T\right)$. Since $\iota(A(\mu))=A(\mu)$ is dense in $\left(\bar{c}\left((A(\mu), \tau)^{-}\right), T\right)=L \oplus D$, it follows that $A(\mu) \cap L$ is a dense subgroup of $L$ with respect to the Euclidean topology of $L$. By $A(\mu) \cap L \subset E \cap L \subset L$, we obtain $L=\overline{A(\mu) \cap L} \subset \overline{E \cap L} \subset E$ since the finite-dimensional subspace $E \cap L$ is complete with respect to $\sigma\left(\left(E^{\prime}\right)^{a}, E^{\prime}\right)$. We denote $\left\{x_{i}\right\}=A(\mu) \cap D$. Then it holds that $A(\mu)=A(\mu) \cap L+\left\{x_{i}\right\}$. We prove that the subgroup $G=A(\mu) \cap L$ is thick in $L$ with respect to the Euclidean topology of $L$. In fact, for every Baire subset $C$ in $L$ satisfying $G \cap C=\phi,(\bar{\ell})^{-1}(C)$ is a Baire subset of $(A(\mu), \tau)^{-}$ and $(\bar{\iota})^{-1}(C) \cap A(\mu)=\phi$. By the thickness of $(A(\mu), \tau)$, it holds that $\bar{\lambda}\left((\bar{\iota})^{-1}(C)\right)=0$, where $\bar{\lambda}$ is the Haar measure on $(A(\mu), \tau)^{-}$. Remark that the Haar measure on $\left(\bar{\imath}\left((A(\mu), \tau)^{-}\right), T\right)=L \oplus D$ coincides with the image measure $\bar{\imath}(\bar{\lambda})$ up to a constant factor. Furthermore, the Haar measure on $L$ is the restriction $\bar{l}(\bar{\lambda}) \mid L$ since $L$ is an open and closed subgroup in $\left(\bar{l}\left((A(\mu), \tau)^{-}\right), T\right)$. Thus we have proved that for every Baire subset $C$ in $L$ with $G \cap C=\phi$, the Haar measure of $C$ is zero, which shows the thickness of $G$ in $L$. This completes the proof.

\section{§4. Quasi-invariant Set for Borel Measure}

Let $E$ be a Souslin locally convex Hausdorff space and $\mu$ be a probability measure on the Borel $\sigma$-algebra $B(E) .(E, B(E))$ is a measurable group since the product $\sigma$-algebra $B(E) \otimes B(E)$ coincides with the Borel $\sigma$-algebra on the product space $E \times E$, see Schwartz [8], Part I, Chapter II. Moreover, $\mu$ is a Radon measure, that is, for every Borel subset $A \in B(E), \mu(A)=\sup \{\mu(K): K$ is compact and $K \subset A\}$, see Schwartz [8], Part I, Chapter II, $\$ 3$.

Lemma 7. $A(\mu)$ is a Borel subset.

Proof. We shall give a sketch of the proof. For details, see Okazaki [7], Proposition 12. For every Borel subset $B \in B(E)$, the function $\mu(B-x)$ in $x$ is Borel measurable since $E$ is Souslin and $\mu$ is Radon. Let $M(E)$ be the set of all probability measures on $E$. We consider the $\sigma$-algebra $\mathscr{M}$ on $M(E)$ generated by $\nu \rightarrow \mathcal{\nu}(B), B \in B(E)$, that is, $\mathscr{M}$ is the minimal $\sigma$-algebra on $M(E)$ which makes each $\nu \rightarrow \nu(B), B \in B(E)$, measurable. Then the mapping $\Psi:(E, B(E)) \rightarrow(M(E) \times$ $M(E), \mathscr{M} \otimes \mathscr{M}), \Psi(x)=\left(\mu_{x}, \mu\right)$, is measurable. By Dubins and Freedman [2], 2.11, the set $D=\{(\xi, \eta) \in M(E) \times M(E): \xi \sim \eta\}$ belongs to $\mathscr{M} \otimes \mathscr{M}$ (here we use the fact that $B(E)$ is countably generated, see Schwartz [8], Part I, Chapter II, $\S 1$, Corollary of Lemma 18). Hence we obtain $A(\mu)=\Psi^{-1}(D)$ belongs to $B(E)$.

Theorem 2. Let $E$ be a Souslin locally convex Hausdorff space and $\mu$ be a 
probability measure on $(E, B(E))$. Suppose that $\mu(A(\mu))>0$. Then

(1) $A(\mu)$ is a locally compact $\sigma$-compact topological subgroup of $E$ with respect to the induced topology from $E$,

(2) the restriction $\nu=\mu \mid A(\mu)$ is equivalent to the Haar measure of $A(\mu)$, and

(3) there exist a finite-dimensional subspace $L \subset E$ and a countable subgroup $\left\{x_{\imath}\right\}$ of $E$ such that $A(\mu)=\bigcup_{\imath=1}^{\infty}\left(L+x_{\imath}\right)$.

Proof. By Lemma 7, $A(\mu)$ is a Souslin topological group with respect to the induced topology from $E$, see Schwartz [8], Part I, Chapter II, §1, Theorem 3. The restriction $\nu=\mu \mid A(\mu)$ is a quasi-invariant Radon measure on $A(\mu)$ with respect to the induced topology from $E$. Furthermore, $(A(\mu), B(A(\mu)), \nu)$ is a separated measurable group, see Lemma 3. Thus by Mackey [6], Lemma 7, Umemura [10], Proposition 6.2 and Yamasaki [12], Part B, Chapter 1, §1, Theorems 1.4 and 1.1, there exists a $\sigma$-finite invariant Radon measure $\lambda$ which is equivalent to $\nu$, see Lemma 4 . Hence by Gowrisankaran [3], $A(\mu)$ is a locally compact $\sigma$-compact topological group with respect to the induced topology from $E$ and $\lambda$ is the Haar measure of $A(\mu)$ up to a constant factor. The $\sigma$-compactness follows by the $\sigma$-finiteness of the Haar measure. By the structure theorem of a locally compact $\sigma$-compact abelian topological group, $A(\mu)$ is isomorphic with $R^{n} \oplus Z^{d} \oplus K$ by Hewitt and Ross [5], Theorem (9.8) and Weil [11], $\S 29$, see the proof of Theorem 1 . Since $K$ is a compact subgroup of the vector space $E$, it follows that $K=\{0\}$. Let $\phi: R^{n} \oplus Z^{d} \rightarrow A(\mu)$ be an isomorphism. Then $\phi$ is linear on $R^{n}$. We put $L=\psi\left(R^{n}\right)$ and $\left\{x_{2}\right\}=\dot{\psi}\left(Z^{d}\right)$. Then $L$ is a finite-dimensional subspace and $A(\mu)=\bigcup_{\imath=1}^{\infty}\left(L+x_{\imath}\right)$. This completes the proof.

\section{References}

[1] Bourbaki, N., Élóments de Ratinenatique Topologie Générale Chapitre 1 et 2, Hermann, Paris, 1965.

[2] Dubins, L. and Freedman, D., Measurable sets of measures, Pacific J, Math., 14 (1964), 1211-1222.

[3] Gowrisankaran, C., Radon measures on groups, Proc. of A.M.S., 25 (1970), $381-$ 384 .

[4] Halmos, P.R., Measure theory, Van Nostrand Reinhold Comp., New York, 1969.

[5] Hewitt, E. and Ross, K.A., Abstract harmonic analysis, Grundlehren der Math. Wiss. 115, Springer-Verlag, Berlin-Heidelberg-New York, 1963.

[6] Mackey, G.W., Borel structure in groups and their duals, Trans. Amer. Math. Soc., 8 (1957), 136-165.

[7] Okazaki, Y., Admissible translates of measures on a topological group, Mem. Fac. Sci. Kyushu Univ., Ser. A, 34 (1980), 79-88.

[8] Schwartz, L., Radon measure on arbitrary topological spaces and cylindrical measures, Tata Inst. of Fund. Res. and Oxford Univ. Press, 1973.

[9] Skorohod, A.V., Integration in Hilbert space, Erzebnisse der Math., 79, SpringerVerlag, Berlin-Heidelberg-New York, 1974.

[10] Umemura, Y., Measures on infinite dimensional vector spaces, Publ. R.I.M.S., 
Kyoto Univ., $\mathbb{1}$ (1965), 1-47.

[11] Weil, A., L'integration dans les groupes topologiques et ses applications, Hermann, Paris, 1965.

[12] Yamasaki, Y., Measures on infinite dimensional spaces, World Scientific, SingaporePhiladelphia, 1985. 